\title{
Performance Analysis of Spatially Multiplexed MIMO using STBC Antenna Diversity Technique
}

\author{
*V.B.Bagde ${ }^{1}$, C.G.Dethe ${ }^{2}$ \\ ${ }^{I}$ Electronics \& Communication Engineering Priyadarshini Institute of Engg. \& Technology, Nagpur, India \\ ${ }^{2}$ Electronics \& Communication Engineering Academic Staff College, RTMNU, Nagpur, India
}

\begin{abstract}
A new wireless broadband technology, MIMO (multiple input multiple output) systems, has gained great popularity for faster data transmission speed and have been actively investigated and successfully deployed for the emerging broadband wireless access networks. Even when a wireless channel with high channel capacity is known, we still require to find superior techniques to achieve high speed data transmission or high reliability. Multiple antenna techniques can be broadly classified into two categories: diversity techniques and spatialmultiplexing techniques. The diversity techniques intend to receive the same information-bearing signals in the multiple antennas or to transmit them from multiple antennas, in so doing improving the transmission consistency. A basic idea of the diversity techniques is to convert Rayleigh fading wireless channel into more stable AWGN-like channel without any catastrophic signal fading. When the spatial-multiplexing techniques are used, the maximum achievable transmission speed can be the same as the capacity of the MIMO channel; however, when the diversity techniques are used, the achievable transmission speed can be much lower than the capacity of the MIMO channel. Spatially multiplexed MIMO (SM-MIMO) systems with STBC antenna diversity technique can transmit data at a higher speed. Signal detection at the receiver side is a thought provoking task for SM MIMO systems. This paper addresses the performance of maximum likelihood detection, zero forcing algorithm and MMSE with successive interference cancellation using different methods of detection for SM MIMO systems.
\end{abstract}

Keywords: diversity, space time block coding, Spatially multiplexed MIMO, maximum likelihood detection.

\section{INTRODUCTION}

In the MIMO system which employs multiple antennas in the transmitter and/or receiver, the correlation between transmit and receive antenna is an important aspect of the MIMO channel. MIMOOFDM is a key technology for nextgeneration cellular communications. Wireless communication systems performance is mainly governed by the wireless channel environment. In fact, the understanding of wireless channels will lay the foundation for the development of high performance and bandwidth-efficient wireless transmission technology .As opposed to the typically static and predictable characteristics of a wired channel, the wireless channel is relatively active and irregular, which makes a precise investigation of the wireless communication system often difficult. In recent years, optimization of the wireless communication system has become vital with the rapid growth of mobile communication services and emerging broadband mobile Internet access services.

Compared to a usual single antenna structure, the channel capacity of a many antenna system with NT transmit and NR receive antennas can be increased by the factor of min (NT; NR), without using additional transmit power or spectral bandwidth. Owing to the forever growing demand of rapid data transmission speed in the recent or future telecommunication systems, the multiple antenna systems have been actively investigated [21] and successfully deployed for the emerging broadband wireless access networks (e.g., Mobile WiMAX) [3]. Even when a wireless channel with high channel capacity is known, superior techniques to achieve high-speed data transmission or high consistency are required. Multiple antenna techniques can be broadly classified into two categories: diversity techniques and spatial-multiplexing techniques [13].The diversity techniques intend to receive the same information-bearing signals in the multiple antennas or to transmit them from multiple antennas, in so doing improving the transmission reliability $[1,3]$. A basic idea of the diversity techniques is to convert Rayleigh fading wireless channel into more stable AWGN-like channel without any catastrophic signal fading. In the spatial-multiplexing techniques, on the other hand, the multiple independent data streams are simultaneously transmitted by the multiple transmit antennas, thereby achieving a higher transmission speed.

The maximum achievable transmission speed can be the same as the capacity of the MIMO channel for the spatial multiplexing techniques used; 
however, when the diversity techniques are used, the achievable transmission speed can be much lower than the capacity of the MIMO channel [27]. Diversity gain can be achieved by space time block coding at the transmitter side which require simple linear processing in the receiver side for decoding $[1,3]$. The performance of linear detection can be improved by ordered successive interference cancellation (OSIC) method without increasing the complexity significantly. It is a bank of linear receivers, each of which detects one of the parallel data streams, with the detected signal components successively cancelled from the received signal at each stage.

\section{SYSTEM MODEL}

Linear signal detection method treats all transmitted signals as interferences except for the desired stream from the target transmit antenna. As a result interference signals from additional transmit antennas are minimized or nullified in the course of detecting the desired signal from the target transmit antenna. The standard linear detection methods include the zero-forcing ( $\mathrm{ZF})$ technique, the minimum mean square error (MMSE) technique, ordered successive interference cancellation (OSIC) and ML (Maximum Likelihood) detection method. The ML detection method outperforms the other detection methods [28]. The spatially multiplexed user data and the equivalent received signals are represented by

$$
\begin{gathered}
x=\left[x_{1}, x_{2}, \ldots \ldots x_{N_{T}}\right]^{T} \\
y=\left[y_{1}, y_{2}, \ldots \ldots \ldots y_{N_{R}}\right]^{T}
\end{gathered}
$$

$\mathrm{x}_{\mathrm{i}}$ and $\mathrm{y}_{\mathrm{j}}$ are transmit signal from $\mathrm{i}^{\text {th }}$ transmit antenna and $\mathrm{j}^{\text {th }}$ received antenna

$$
\begin{gathered}
y=H_{x}+z \\
=h_{1} x_{1}+h_{2} x_{2}+\ldots \ldots+h_{N_{T}} x_{N_{T}}+z
\end{gathered}
$$

Where $\mathrm{H}=$ channel matrix

$z=$ Guassian noise

$$
z=\left[z_{1}, z_{2}, \ldots \ldots z_{N_{R}}\right]^{T}
$$

To facilitate the detection of desired signals from each antenna, the effect of the channel is inverted by a weight matrix W such that

$$
\tilde{x}=\left[\tilde{x}_{1} \tilde{x}_{2} \ldots \ldots \tilde{x}_{N_{T}}\right]^{T}=W_{y}
$$

The detection of each symbol is given by linear combination of the received signals.

\section{The system model is shown in Fig.1}

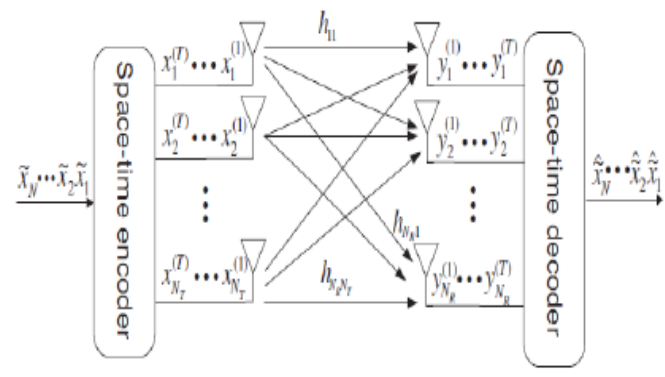

Fig.1. Space Time Coded MIMO System

that is, detection of each symbol is given by a linear combination of the received signals.

\section{ML SIGNAL DETECTION}

Maximum likelihood (ML) detection calculates the Euclidean distance between the received signal vector and the product of all possible transmitted signal vectors with the given channel $\mathrm{H}$, and finds the one with the least distance. Let $\mathrm{C}$ and NT represent a set of signal constellation symbol points and a number of transmit antennas, respectively. Then, ML detection determines the estimate of the transmitted signal vector $\mathrm{x}$ as

$$
\tilde{x}_{M L}=\underset{x \in C^{N_{T}}}{\arg \min }\left\|y-H_{x}\right\|^{2}
$$

Where $\left\|y-H_{x}\right\|^{2}$ corresponds to ML metric

The ML detection outperforms the other detection methods like zero forcing technique, ordered successive interference cancellation OSIC Signal Detection, and MMSE ( minimum mean square error) technique [28].

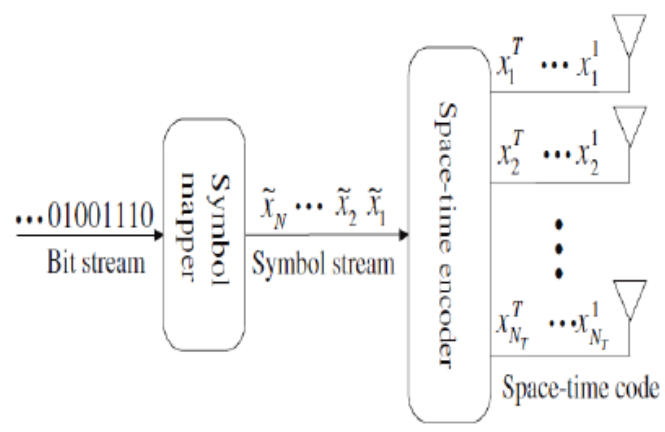

Fig.2. Space-Time Block Encoder 


$$
\begin{gathered}
h_{1}(t)=h_{1}\left(t+T_{S}\right)=h_{1}=\left|h_{1}\right| e^{j \theta_{1}} \\
h_{2}(t)=h_{2}\left(t+T_{S}\right)=h_{2}=\left|h_{2}\right| e^{j \theta_{2}}
\end{gathered}
$$

Where, magnitude of $h_{i}$ and $\theta_{i}$ denote the amplitude gain and phase rotation over the two symbol periods, $i=1,2$.

Let $\mathrm{y}_{1}$ and $\mathrm{y}_{2}$ denote the received signals at time $\mathrm{t}$ and $\mathrm{t}+\mathrm{Ts}$, respectively, then

$$
\begin{gathered}
y_{1}=h_{1} x_{1}+h_{2} x_{2}+z_{1} \\
y_{2}=-h_{1} x_{2}^{*}+h_{2} x_{1}^{*}+z_{2}
\end{gathered}
$$

Where, $z_{1}$ and $z_{2}$ are the additive noise at time $t$ and $t+T s$, respectively. Taking complex conjugate of the second received signal, we have the following matrix vector equation:

$$
\left[\begin{array}{l}
y_{1} \\
y_{2}^{*}
\end{array}\right]=\left[\begin{array}{l}
h_{1} h_{2} \\
h_{2}^{*}-h_{1}^{*}
\end{array}\right]\left[\begin{array}{l}
x_{1} \\
x_{2}
\end{array}\right]+\left[\begin{array}{l}
z_{1} \\
z_{2}^{*}
\end{array}\right]
$$

If channel gains, $\mathrm{h} 1$ and $\mathrm{h} 2$, are exactly known to the receiver then the transmit symbols are now two unknown variables in the matrix. Multiplying both sides of Equation by the Hermitian transpose of the channel matrix,

$$
\begin{aligned}
& {\left[\begin{array}{l}
h_{1}^{*} h_{2} \\
h_{2}^{*}-h_{1}
\end{array}\right]\left[\begin{array}{l}
y_{1} \\
y_{2}^{*}
\end{array}\right]=\left[\begin{array}{l}
h_{1}^{*} h_{2} \\
h_{2}^{*}-h_{1}
\end{array}\right]\left[\begin{array}{l}
h_{1} h_{2} \\
h_{2}^{*}-h_{1}^{*}
\end{array}\right]\left[\begin{array}{l}
x_{1} \\
x_{2}
\end{array}\right]+\left[\begin{array}{l}
h_{1}^{*} h_{2} \\
h_{2}^{*}-h_{1}
\end{array}\right]\left[\begin{array}{l}
z_{1} \\
z_{1}^{*}
\end{array}\right]} \\
& \left(\left|h_{1}\right|^{2}+\left|h_{2}\right|^{2}\right)\left[\begin{array}{l}
x_{1} \\
x_{2}
\end{array}\right]+\left\lfloor\begin{array}{l}
h_{1}^{*} z_{1}+h_{2} z_{1}^{*} \\
h_{2}^{*} z_{1}-h_{1} z_{1}^{*}
\end{array}\right]
\end{aligned}
$$

Subsequent input-output relationships are obtained.

$$
\left[\begin{array}{c}
\widetilde{y}_{1} \\
\widetilde{y}_{2}
\end{array}\right]=\left(\left|h_{1}\right|^{2}+\left|h_{2}\right|^{2}\right)\left[\begin{array}{l}
x_{1} \\
x_{2}
\end{array}\right]+\left[\begin{array}{c}
\widetilde{z}_{1} \\
\widetilde{z}_{2}
\end{array}\right]
$$

Where

$$
\left[\begin{array}{l}
\widetilde{y}_{1} \\
\widetilde{y}_{2}
\end{array}\right] \cong\left[\begin{array}{l}
h_{1}^{*} h_{2} \\
h_{2}^{*}-h_{1}
\end{array}\right]\left[\begin{array}{l}
y_{1} \\
y_{2}^{*}
\end{array}\right]
$$

And

$$
\left[\begin{array}{l}
\widetilde{z_{1}} \\
\widetilde{z_{2}}
\end{array}\right] \cong\left[\begin{array}{l}
h_{1}^{*} h_{2} \\
h_{2}^{* 1}-h_{1}
\end{array}\right]\left[\begin{array}{l}
z_{1} \\
z_{1}^{*}
\end{array}\right]
$$

Other antenna interference does not exist any longer, that is, the unnecessary symbol $\mathrm{x} 2$ dropped out of $y 1$, while the unwanted symbol $\mathrm{x} 1$ dropped out of y2.

$$
\widetilde{x}_{i, M L}=Q\left(\frac{\widetilde{y}_{i}}{\left|h_{1}\right|^{2}+\left|h_{2}\right|^{2}}\right), i=1,2 .
$$

Where $\mathrm{Q}$ (.) denotes a slicing function that determines a transmit symbol for the given constellation set. The above equation implies that $\mathrm{x} 1$ and $\mathrm{x} 2$ can be decided separately, which reduces the decoding complexity of original ML decoding algorithm.

\section{SIMULATION RESULTS}

Simulations are carried out in Mat lab. Performance based on two, four and eight transmit and receive antennas showing BER for BPSK and QPSK modulation in a Rayleigh fading channel with Alamouti Space Time Block Coding is shown in the following simulation results.

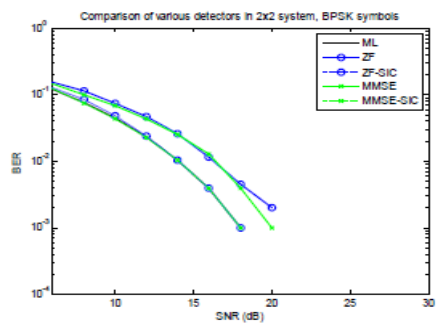

Fig 4. Performance of 2 X 2 BPSK Scheme

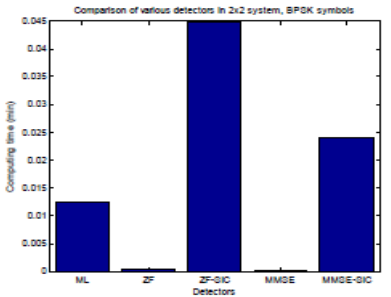

Fig 5. Computational time of 2 X 2 BPSK Scheme

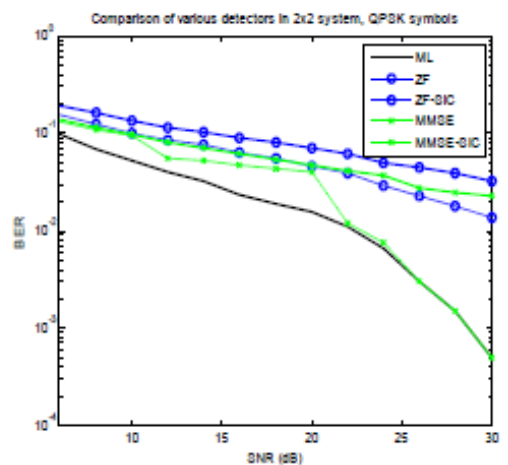

Fig.6. Performance of $2 \times 2$ QPSK Scheme 


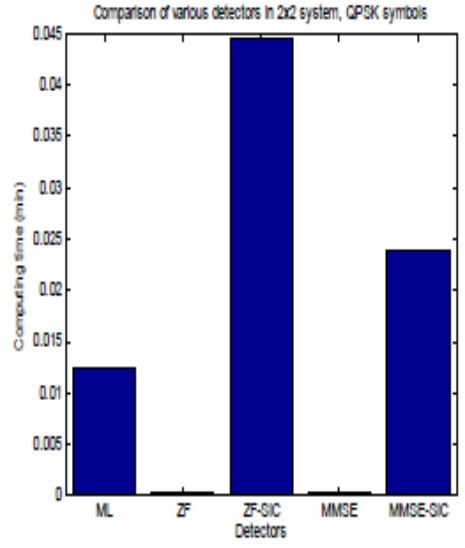

Fig.7. Computational time of $2 \times 2$ QPSK Scheme

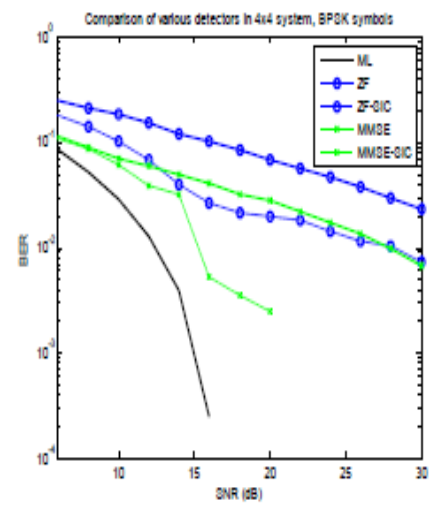

Fig.8. Performance of 4X 4 BPSK Scheme

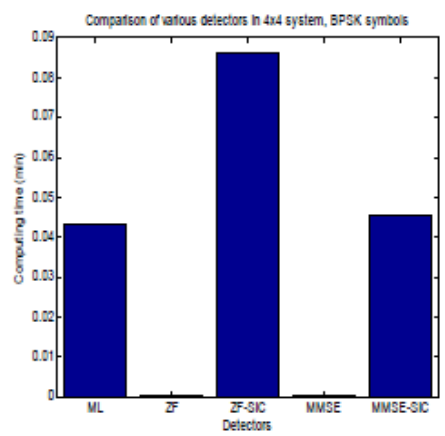

Fig.9. Computational time of $4 \mathrm{X} 4 \mathrm{BPSK}$ Scheme

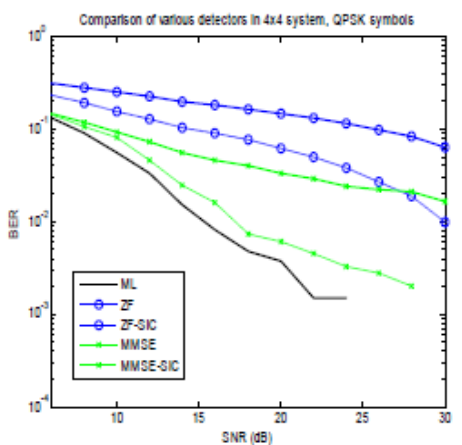

Fig.10. Performance of $4 \times 4$ QPSK Scheme

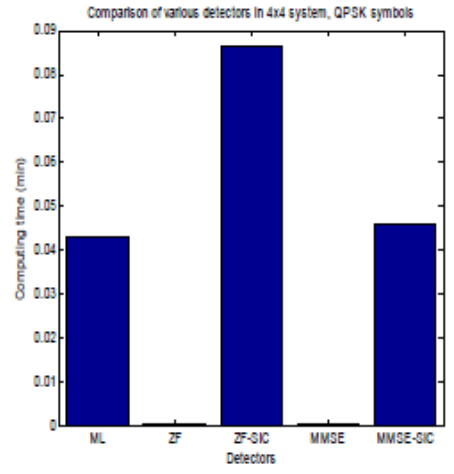

Fig.11. Computational time of $4 \times 4$ QPSK Scheme

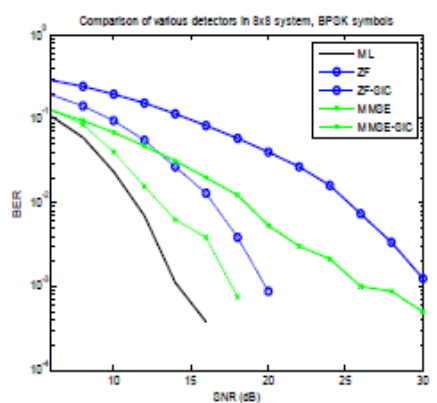

Fig.12. Performance of $8 \times 8$ BPSK Scheme

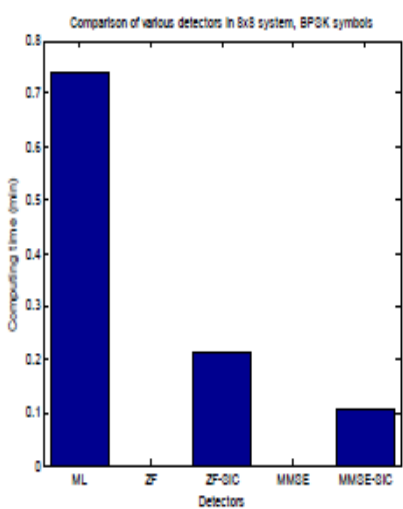

Fig.13. Computational time of $8 \times 8$ BPSK Scheme

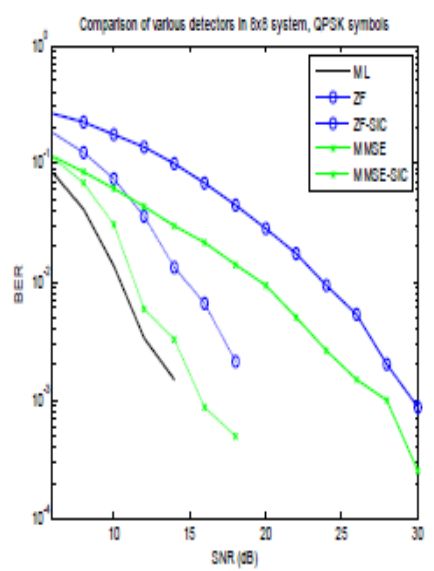

Fig.14. Performance of $8 \times 8$ QPSK Scheme 


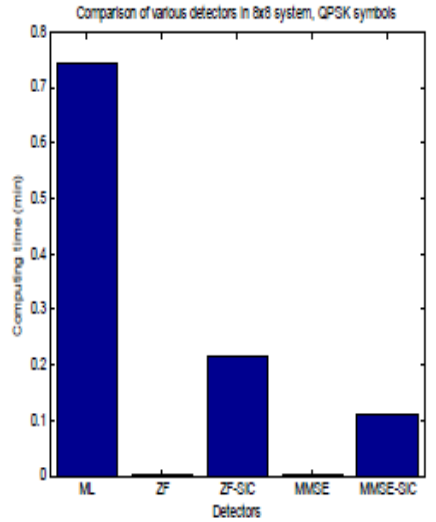

Fig.15. Computational time of $8 \times 8$ QPSK Scheme

\section{CONCLUSION}

Transmit diversity techniques have been widely adopted since it reduces processing complexity of the receiver and require multiple antennas only on the transmitter side. Spatially multiplexed MIMO (SM-MIMO) systems can transmit data at a higher speed than MIMO systems using antenna diversity. However, spatial demultiplexing or signal detection is efficiently carried out by using ML detection technique. The ML detection outperforms the OSIC detection. Therefore, there have been active researches to develop the detection methods that still consider the ML detection method while still achieving a near-optimal performance with less complexity.

\section{REFERENCES}

[1] S.M.Alamouti, "A Simple Transmit Diversity Technique for Wireless Communication,” IEEE J Sel. Areas Commn.vol 16, no.8,pp 1451- 1458,Oct 1998.

[2] A.F.Naguib, N. Sheshadri and A.R.Calderbank, "Applications of space time block Codes and interference suppression for high capacity and high data rate wireless Systems," in Proc 32 th Ann. Asilomar Conf.Signals,Syst. Comput ,Pacific Grove, CA, Nov.1998, pp-18031810.

[3] V.Tarokh, N.Sheshadri and A.R.Calderblank "Space time codes for high data rate wireless communication: Performance criterion and code construction", IEEE Trans Inf. Theory., vol 44, no.2,pp 744765,March 1998.

[4] J.H.Winters, J Salz, and R.D.Gitlin "The impact of antenna diversity on the capacity of wireless communication systems", IEEE Trans Commn.,vol 42, no.2, pp.1740-1751, Feb-Mar-April.1994.
[5] S.N.Diggavi,N,AL Dahir",A Stamoulis and A,R,Calderbank "Great expectations : The value of spatial diversity to wireless networks" ,'Proc.IEEE,VOL 92 , no.2,pp.219-270,Feb 2004.

[6] J.Li, K.B.Lateif and Z.Cao "Co-channel Interference Cancellation for space time coded OFDM Systems",IEEE Trans Wireless Commn. vol.2, no.1, pp.41-49, Jan 2003.

[7] W.Su and X.Zia, "Quasi Orthogonal space time block codes with full diversity", IEEE Trans Inf Theory, vol 50, no.10, pp.23312347, Nov 2004

[8] H.Trigui and D.T.M.Slock, "Performance bounds for co-channel interference cancellation within the current GSM standard”, in Signal Processing ,New York : Elsevier ,2000,vol 80,pp.1335- 1346.

[9] J.G.Proakis, Digital Communications, 3rd ed.New York: McGrawhill, 1995.

[10] Pascal Chevalier and Florian Dupuy, "Widely Linear Alamouti Receiver for the Reception of Real -Valued Constellations Corrupted by Interferences- The Alamouti SAIC/MAIC Concept,"

[11] A.Wiittneben, "Base station modulation diversity for digital SIMULCAST", in Proc 1991 IEEEVehicular Technology Conf.(VTC 41 st ),May 1991,pp 848-853.

[12] "A new bandwidth efficient transmit antenna modulation diversity scheme for linear digital Modulation", in Proc.1993 IEEE International Conf. Communications (ICC; 93),May 1993,pp 1630 - 1634.

[13] J.H.Winters, "The diversity gain of transmit diversity in wireless systems with Rayleigh fading,"inProc. 1994

ICC/SUPERCOMM.New Orleans,LA,May 1994 ,vol 2 ,pp 1121- 1125.

[14] G.J. Foschini and M.J.Gans, "On limits of Wireless communications in a fading Environment when using multiple antennas," Wireless Pers. Commun, vol.6, no.3, pp.311 -335,1998.

[15] V, Tarokh, H.Jafarkhani, and A.R.Calderbank, "Space Time block codes from orthogonal designs," IEEE Trans.Inf. Theory, vol.45, pp.1456-1467, Jul.1999.

[16] [V.Tarokh ,A.Naquib ,N.Sheshadri, and A,R Calderbank, "Combined array processing and space time coding ", IEEE Trans .Inf.Theory, vol.45, no.4,pp.1121-1128,May 1999.

[17] H.Jafarkhani, 'A quasi-orthogonal space time block code," IEEE Trans.Commn, vol.49, no.1 Jan 2001. 
[18] R.Meyer, W.H.Gerstacker, R.Schober, and J.B.Huber, "A single antenna interference cancellation algorithm for increased GSM capacity," IEEE Trans Wireless Commn., Vol.5,no.7,pp.1616 1621, Jul.2006.

[19] M.Austin, "SAIC and synchronized networks for increased GSM capacity," $3 \mathrm{~g}$ Americas' SAICWorking Group Sept.2003.

[20] B. Picinbono and P.Chevalier, "Widely Linear estimation with complex data," IEEE Trans.Signal Process. vol.43, no.8, pp.20302033, Aug.1995.

[21] G.J.Foschini, "Layered space -time architecture for wireless communication in a fading environment when using multielementantennas," Bell Labs Tech.J., vol.1,pp.41-59, 1996.

[22] J.H.Winters, J.Salz, and R.D.Gitlin, "The capacity increase of wireless systems with antenna diversity," in Proc.1992 Conf.Inform.Sciences Systems., Princeton,NJ, Mar,18-20,1992.

[23] D.J.Goodman, "Trends in cellular and cordless communications." IEEE Communications Mag., vol.29, pp.31-40, June 1991.
[24] Y.Li, AND Scollenberger, "Adaptive antenna array for OFDM systems with co channel Interferences," IEEE Trans.Commun, vol.47, pp 211- 229, Feb 1999.

[25] J.H.Winters, "Smart antennas for wireless systems." IEEE Pers.Commn Mag., pp.2327.1998.

[26] Long Shi, , Wei Zhang, , and Xiang-Gen Xia, "Space-Time Block Code Designs for Two-User MIMO X Channels" IEEE Transactions on Communication, Vol. 61, NO. 9, Sept 2013

[27] Sanhdu, S. and Paulraj, A. (2000) Spacetime block codes: a capacity perspective. IEEE Commun. Letters, 4(12),384-386.

[28] Kim, J., Kim, Y., and Kim, K. (2007) Computationally efficient signal detection method for next generation mobile communications using multiple antennas. SK Telecommun. Review, 17(1C), 183191.

[29] Wookbong Lee, Inkyu Lee, Jim Sam Kwak, Bin-Chul Ihm,Shuangfeng Han , Multi BS MIMO Cooperation : Challenges and Practical Solutions in 4G Systems."IEEE Wireless Communications, Feb 2012

\footnotetext{
International Journal of Engineering Research and Applications (IJERA) is UGC approved Journal with Sl. No. 4525, Journal no. 47088.

V.B .Bagde. "Performance Analysis of Spatially Multiplexed MIMO using STBC Antenna Diversity Technique." International Journal of Engineering Research and Applications (IJERA) 7.7 (2017): 45-50.
} 